\title{
EEG Sleep in Cushing's Disease and Cushing's Syndrome: Comparison with Patients with Major Depressive Disorder
}

\author{
James E. Shipley, David E. Schteingart, Rajiv Tandon, \\ Atul C. Pande, Leon Grunhaus, Roger F. Haskett, and \\ Monica N. Starkman
}

Because patients with Cushing' syndrome (CS) and Major depressive disorder (MDD) share features of hypercortisolism and the depressive syndrome, we compared electroencephalographic (EEG) sleep in patients with pituitary-ACTH-dependent Cushing's syndrome (Cushing's disease, CD), patients with ACTH-independent Cushing's syndrome (AICS), patients with major depressive disorder (MDD), and normal subjects. There were substantial similarities in the abnormal polysomnography profiles of patients with $C D, A I C S$, and MDD. All three patient groups demonstrated poorer sleep continuity, shortened rapid eye movement (REM) latency, and increased first REM period density compared with normal subjects. In addition, AICS patients and MDD patients had elevated REM activity and density. These findings are discussed in terms of models of pathophysiology that relate abnormalities in sleep, mood, and hypothalamic-pituitary-adrenal function.

\section{Introduction}

Many patients with spontaneous Cushing's syndrome (CS), which is characterized by hypersecretion and loss of circadian rhythm of cortisol, manifest features of the depressive syndrome including depressed and irritable mood, cognitive difficulties, and vegetative complaints (Starkman 1987; Starkman et al 1981; Haskett 1985). Symptoms of disordered sleep are common, with middle-night awakenings and early morning awakening observed in over 50\% of patients (Starkman et al 1981).

Pioneering studies of electroencephalographic (EEG) sleep in patients with CS were done nearly 2 decades ago (Krieger and Glick 1974; Krieger et al 1976), and remain the only such studies in the literature. These investigators studied five patients with pituitaryACTH-dependent CS, which is Cushing's disease (CD), and six patients with ACTH-

From the Sleep (JES), Consultation-Liaison (MNS), Schizophrenia (RT), and Depression (ACP, LG, RFH) Programs, Department of Psychiatry; and the Division of Endocrinology and Metabolism (DES), Department of Medicine, University of Michigan School of Medicine, Ann Arbor, MI.

Address reprint requests to James E. Shipley, M.A., M.D., Med Inn Box 0840, 1500 East Medical Center Drive, Ann Arbor, MI 48109-0840.

Received May 20, 1991; revised January 29, 1992. 
independent CS (AICS) due to a primary adrenal adenoma. They observed poorer sleep continuity with increased awake time and a diminished percentage of slow wave (deltaठ) sleep.

Since that time, a significant proportion of psychiatric patients with major depressive disorder (MDD) have been shown to manifest dysregulation of the hypothalamic-pituitaryadrenal (HPA) axis, including elevated cortisol levels and early escape from suppression by dexamethasone (Carroll et al 1981; Stokes et al 1984; Evans and Nemeroff 1987). Abnormalities in sleep continuity and a decreased percentage of $\delta$ sleep have been described in MDD patients as well (Reynolds and Kupfer 1987). In addition, rapid eye movement (REM) sleep has consistently been shown to be abnormal in MDD, particularly shortened REM latency and increased REM density (Kupfer and Ehlers 1989).

Because patients with CS and patients with MDD share features of HPA axis dysregulation and the depressive syndrome, direct comparison of their sleep EEG profiles may help shed light on the pathophysiological mechanisms common to both conditions. We therefore extended the pioneering, small-sample studies of EEG sleep in patients with $C D$ by adding an assessment of features of REM sleep, and compared CD patients directly with an age-matched group of patients with MDD.

\section{Methods}

\section{Subjects}

CS Subjects. A consecutive series of patients with suspected CS were admitted to the Clinical Research Center (CRC) of the Universily of Michigan Hospital for diagnostic evaluation. The diagnosis of CS was established by standard clinical criteria, for example, moon facies, truncal obesity, skin and muscle atrophy, and most, if not all, of the following findings related to cortisol: lack of normal circadian rhythm in association with excessive secretion as measured by high urinary-free cortisol, cortisol secretion rates, and plasma cortisol values. Patients were further classified according to the type of CS. Patients with CD had normal or elevated ACTH levels, failed to suppress cortisol normally after $2 \mathrm{mg}$ of dexamethasone, but showed greater than $50 \%$ suppression after $8 \mathrm{mg}$. The diagnosis of $\mathrm{CD}$ was further confirmed through microscopic pathology following pituitary surgery. Patients with AICS had suppressed ACTH levels and failed to suppress cortisol after 8 $\mathrm{mg}$ of dexamethasone. The adrenal pathology was confirmed after surgery.

Protocol for CS Subjects A semistructured psychiatric interview was done (MNS) during the baseline period (Starkman et al 1981). A 14-item Hamilton Rating Scale fur Depression (HAM-D, modified for exclusion of items 15-17 relevant to their medical illness) was obtained (Starkman et al 1986). The mean \pm SD rating was $8.7 \pm 5.6$ (range 2-17).

Sleep studies were carried out in the CRC on the night of admission and the following night. Of 29 patients ultimately determined to have CS, 14 were excluded from anilysis of sleep stages from night 2 for the following reasons: seven had significant sleep apnea on night 1 ( $>9$ respiratory events/hr); two had taken within the prior 2 weeks medications that would invalidate the sleep measures (an antidepressant and a hypnotic), four had intermittent rather than chronic cortisol hypersecretion, and one svas older than 70 years. Our sample for analysis of sleep architecture on night 2 thus consisted of 11 patients with CD two men, nine women) and four patients (one man, three women) with AICS. Nine 
of these $11 \mathrm{CD}$ patients received antihypertensive medications, but none were receiving alpha-methyl dopa. Five received diuretics, two received clonidine, one received the betablocker metoprolol, two received calcium channel blockers (nifedipine, verapamil), one an angiotensin converting enzyme (ACE) inhibitor (enalapril), and one prazosin.

Depressed Patients. Patients with definite MDD by Research Diagnostic Criteria (RDC) and a 17-item Hamilton Depression (HAM-D) score $\geqslant 15$ were recruited from the Depression Program of the University of Michigan; nine of these were part of a larger sample used in a preliminary report of the association of postdexanethasone cortisol level with EEG sleep measures (Shipley et al 1989). Patients were matched for age ( \pm 3 years) to the $11 \mathrm{CD}$ patients. These MDD patients included eight women and three men (nine inpatients and two outpatients). RDC subtypes showed one to be psychotic; four were endogenous; one was bipolar I and one bipolar II; and eight were primary and three secondary. The mean \pm SD 17-item HAM-D was $19.3 \pm$ 5.0. Six were dexamethasone suppression test (DST) nonsuppressors to $1 \mathrm{mg}$ of dexamethasone. Patients were psychotropic drug free for a minimum of 14 days. Inpatient rooms were connected by cable to the sleep laboratory. Recordings were obtained for 2 consecutive nights, and data from the second night (nine inpatients) or third night (two outpatients) were used for the analysis.

Normal Subjects. Eleven healthy volunteers (six men, five women) were recruited from advertisements, screened for medical or psychiatric illness, and matched for age to the $C D$ patients (nine were $\leqslant 4$ years and two were $\leqslant 5$ years). No one was taking medication that would affect the sleep measures, and each was asked to abstain from alcohol for the 2 weeks preceding the recordings. Subjects slept for 2 consecutive nights in rooms in the sleep laboratory, and sleep apnea was ruled out on night 1 .

\section{Polysomnography}

Data were transmitted from the CS patienis' bedside to the sleep laboratory using a TeleDiagnostics S10/R 10 telephone telemetry system, and sleep tracings were recorded using a Grass Model 78D polygraph, yielding a nominal EEG band pass of $0.3-30 \mathrm{~Hz}$. Sleep recordings for normal controls used a Telefactor cable telemetry system and a Grass model 78D polygraph, yielding a nominal EEG band pass of $0.5-30 \mathrm{~Hz}$. Sleep recordings for MDD patients were identical to the latter in two cases and for the remainder used remote direct cable connection to a Grass model 78D polygraph yielding a nominal EEG band pass of $0.3-30 \mathrm{~Hz}$. For all subjects, electrode impedances were less than 5,000 ohms and paper speed was $10 \mathrm{~mm} / \mathrm{sec}$. On the first night of hospitalization for controls and CS patients a full montage polysomnogram was used to rule out primary sleep disorders such as sleep apnea or nocturnal myoclonus. This included EEG (C3/A2), referential electrooculogram (EOG), submental electromyogram (EMG), respiratory monitoring (nasal-oral thermistors, abdominal and chest strain gauges, and ear oximetry), electrocardiogram, and EMG of the anterior tibialis muscle. Most patients underwent a second recording night and next night, in which information required for sleep staging was recorded (EEG, EOG, and chin EMG).

All polygraph records were scored visually using a $60 \mathrm{sec}$ epoch according to modified Rechtshaffen et al (1968) criteria. The visually scored EEG sleep variables were divided into three groups: sleep continuity, sleep architecture, and REM sleep indices.

Sleep Continuity Indices. Total recording period (TRP); time spent asleep (TSA) = 
net sleep time; sleep efficiency (TSATRP $\times 100$ ); sleep latency (time from the beginning of the recording until the onset of Stage 2 sleep for at least 10 min interrupted by no more than $2 \mathrm{~min}$ of Stage 1, or $1 \mathrm{~min}$ of stage 1 plus $1 \mathrm{~min}$ of wakefulness); sleep maintenance (percentage of time asleep during the total recording period after sleep latency); wakefulness after sleep onset (time spent awake during the night which is bounded by sleep); and wakefulness during the last $2 \mathrm{hr}$ of the recording.

Sleep Architecture Indices. Percentages of net sleep time spent in Stage 1, 2, 3, 4, and REM; and $\delta$, or slow-wave sleep (the sum of Stages 3 and 4).

REM Sleep Indices. REM time (RT); REM activity (RA, an integrative measure of the frequency of REMs scored by visual inspection on a scale of $0-8 / \mathrm{min}$ of REM sleep); REM density (RA/RT); and PEM slcep latency (the time between sleep onset and the first REM period, minus intermittent wakefulness during that interval). A REM period was defined as a minimum of 3 min of REM sleep within 30 min of each other.

\section{Statistical Analysis}

Comparisons for individual sleep variables on night 2 between the three main groups of subjects were done by analysis of variance (ANOVA) with follow-up comparisons using the Fisher Protected LSD test. As the AICS group included only four subjects, these were not included in the ANOVA. Spearman rank order correlations between baseline cortisol measures in the $C D$ patients versus their sleep measures were performed. To determine if urinary-free cortisol (UFC) measures were related to sleep measures in CD patients, median-split analyses was done using Mann-Whitney U-tests.

\section{Results}

Results for the ANOVA for EEG sleep measures for patients with CD, MDD, and normal subjects are presented in Table 1 . Also displayed for comparative purposes are mean \pm SD for sleep data for the four AICS subjects.

Compared with healthy control subjects, the $11 \mathrm{CD}$ patients showed significantly poorer sleep continuity, lighter non-REM sleep, shorter REM latency, and increased REM density in the first REM period. Detailed statistical comparisons between the patients with $C D$ and normal subjects, as well as data concerning the increased incidence of sleep apnea in CS, are given elsewhere (Shipley et al, in press).

Many similarities were observed in the sleep of $C D$ patients and patients with MDD. Both groups showed a significantly longer sleep latency, less total sleep time, and lower sleep efficiency than did the normal subjects. In both CD and MDD patients, REM latency was significantly shortened, and REM density in the first REM period was significantly increased compared with control subjects. The mean percentages of de!ta sleep (sum of Stages 3 and 4) were not significantly diffeient among the groups.

There were also some differences between $\mathrm{CD}$ and MDD patients. $\mathrm{C}$ ) patients differed from both MDD patients and controls in having greater awake time Juring the sleep period and thus lower sleep maintenance, and a significantly greater per centage of Stage 1 sleep (lightest sleep). CD patients did not differ from normal subjects $n$ REM activity, whereas MDD patients had markedly greater REM activity than either normal subjects or CD patients. All-night REM density in CD patients was intermediate between normals 
Table 1. EEG Sleep Measures in CD, MDD, AICS Patients ${ }^{a, b}$ and Normal Controls

\begin{tabular}{|c|c|c|c|c|c|c|c|}
\hline \multirow[b]{2}{*}{ Variables } & \multirow[b]{2}{*}{$\mathrm{CD}$} & \multirow[b]{2}{*}{ MDD } & \multirow[b]{2}{*}{ Normals } & \multirow[b]{2}{*}{ AICS } & \multicolumn{3}{|c|}{$p<$} \\
\hline & & & & & $\begin{array}{c}\text { CD } \\
\text { vs. } \\
\text { MDD }\end{array}$ & $\begin{array}{l}\text { CD vs. } \\
\text { normals }\end{array}$ & $\begin{array}{c}\text { MDD } \\
\text { vs. } \\
\text { normals }\end{array}$ \\
\hline Age & $37.1 \pm 11.9$ & $37.5 \pm 11.4$ & $38.2 \pm 11.7$ & $33.3 \pm 16.7$ & & & \\
\hline $\begin{array}{l}\text { Total recording period } \\
\text { Sleep continuity }\end{array}$ & $409.2 \pm 32.5$ & $409.1 \pm 53.1$ & $413.2 \pm 43.3$ & $412.3 \pm 32.0$ & & & \\
\hline Sleep latency & $50.7 \pm 25.8$ & $34.3 \pm 31.5$ & $13.5 \pm 15.7$ & $18.8 \pm 7.4$ & & 0.001 & 0.01 \\
\hline $\begin{array}{l}\text { Awake during sleep } \\
\text { time }\end{array}$ & $43.5 \pm 34.8$ & $12.5 \pm 22.8$ & $16.4 \pm 17.6$ & $27.8 \pm 15.8$ & 0.01 & 0.05 & \\
\hline Total sleep time & $306.0 \pm 45.8$ & $341.5 \pm 73.6$ & $381.9 \pm 50.8$ & $351.5 \pm 48.9$ & & 0.01 & \\
\hline $\begin{array}{l}\text { Sleep efficiency } \\
(\%)\end{array}$ & $74.7 \pm 7.9$ & $83.2 \pm 14.0$ & $92.3 \pm 6.0$ & $85.0 \pm 7.0$ & & 0.01 & 0.05 \\
\hline $\begin{array}{l}\text { Sleep maintenance } \\
(\%)\end{array}$ & $87.7 \pm 9.5$ & $96.4 \pm 6.2$ & $95.7 \pm 4.7$ & $92.5 \pm 4.8$ & 0.01 & 0.05 & \\
\hline \multicolumn{8}{|l|}{ Sleep architecture } \\
\hline Strge $1 \%$ & $24.7 \pm 13.3$ & $10.1 \pm 10.8$ & $13.0 \pm 5.2$ & $9.6 \pm 2.9$ & 0.01 & 0.05 & \\
\hline Sluge $2 \%$ & $50.8 \pm 12.2$ & $51.4 \pm 12.0$ & $59.7 \pm 8.8$ & $54.9 \pm 16.4$ & & & \\
\hline Stage $3 \%$ & $3.3 \pm 5.0$ & $6.5 \pm 6.0$ & $5.4 \pm 4.7$ & $3.5 \pm 5.4$ & & & \\
\hline Stage $4 \%$ & $2.5 \pm 8.3$ & $3.7 \pm 7.2$ & $2.1 \pm 3.3$ & $3.8 \pm 7.7$ & & & \\
\hline Delta \% & $5.8 \pm 12.5$ & $10.2 \pm 10.6$ & $7.5 \pm 7.4$ & $7.4 \pm 13.0$ & & & \\
\hline REM \% & $18.7 \pm 7.8$ & $28.2 \pm 6.3$ & $19.8 \pm 4.2$ & $28.1 \pm 6.6$ & 0.01 & & 0.01 \\
\hline \multicolumn{8}{|l|}{ REM measures } \\
\hline $\begin{array}{l}\text { REM latency minus } \\
\text { awake }\end{array}$ & $42.3 \pm 34.7$ & $35.6 \pm 18.9$ & $90.2 \pm 54.5$ & $37.0 \pm 13.1$ & & 0.01 & 0.01 \\
\hline REM periods & $3.5 \pm 0.8$ & $3.4 \pm 1.0$ & $3.4 \pm 1.1$ & $3.8 \pm 0.5$ & & & \\
\hline REM activity (U) & $67.7 \pm 42.0$ & $175.0 \pm 57.7$ & $65.5 \pm 28.0$ & $168.3 \pm 34.1$ & 0.001 & & 0.001 \\
\hline REM density & $1.1 \pm 0.4$ & $1.9 \pm 0.5$ & $0.9 \pm 0.3$ & $1.8 \pm 0.6$ & 0.001 & & 0.001 \\
\hline REM 1 time & $15.5 \pm 7.7$ & $26.2 \pm 16.6$ & $21.3 \pm 11.8$ & $23.8 \pm 11.2$ & & & \\
\hline REM I activity & $19.6 \pm 16.7$ & $53.2 \pm 49.2$ & $13.2 \pm 7.6$ & $43.0 \pm 23.6$ & 0.05 & & 0.01 \\
\hline REM 1 density & $1.2 \pm 0.5$ & $1.6 \pm 0.8$ & $0.6 \pm 0.2$ & $1.7 \pm 0.4$ & & 0.05 & 0.001 \\
\hline
\end{tabular}

Sleep measures are mean \pm SD.

$u_{n}=11 /$ group except AICS n $\approx 4$.

${ }^{b}$ ANOVA excludes AICS group.

and MDD patients, although this reached significance only for the first REM period density. Mean Stages 3, 4, and delta sleep were not significantly different among the groups.

Given the significant impairments in sleep continuity, shortening of REM latency, and increase in first REM period density we observed in the patients with $C D$, we investigated the association between the degree of cortisol elevation and the sleep measures in these patients. In the first analysis, a median split was made as a function of baseline UFC: five cases below $300 \mu \mathrm{g} / \mathrm{day}$ (range 69-291), versus six cases above $300 \mu \mathrm{g} / \mathrm{day}$ (range $322-3,000$ ). Mean age did not differ in these two groups. The subgroup with less marked elevation in UFC had higher whole-night REM density $(p<0.05)$, higher REM activity $(p<0.05)$, and higher REM density in the first REM period $(p<0.05)$. In the second type of analysis, Spearman correlation coefficients were computed between baseline plasma cortisol levels (8 AM, 4 PM, and 10 PM) and sleep measures. Cortisol level at 4 PM was positively correlated with awake time during the sleep period $(0.77, p<0.05)$ and negatively with sleep maintenance $(-0.74, p<0.05)$. Mean plasma cortisol was 
negatively correlated with total REM activity $(-0.75, p<0.05)$ and with REM activity in the first REM period $(-0.67, p<0.05)$. In summary, higher plasma cortisol and higher UFC were associated with lower REM activity, and higher plasma cortisol was associated with greater amounts of awake time and consequent lowered sleep maintenance.

\section{Comparison of Sleep Profiles of Two Forms of CS}

AICS and CD are of theoretical interest. AICS patients are exceedingly rare, and our current sample size of four is still too small for statistical analysis. Data for the four patients with AICS are included in Table 1 for comparative purposes, however, and reveal some differences from CD. Inspection of the data reveals that the AICS patients were remarkably similar to the MDD patients. Most notably, sleep continuity for the AICS patients was not as impaired as in CD patients, and REMi activity and density in the AICS patients was higher than in CD patients. The AICS patients were similar to both the $C D$ and MDD patients in that all three groups manifested lowered sleep efficiency, shortened REM latency, and increased first REM period density.

\section{Discussion}

This study reports the results of the first direct comparison of sleep EEG abnormalities in patients with $C D$ and MDD. The results indicate that there are substantial similarities in the abnormal polysomnography profiles of patients with these two disorders. Both groups of patients manifested significantly increased sleep latency and decreased sleep efficiency compared with normal subjects. Both groups manifested significantly shortened REM latency and increased REM density in the first REM period. Some differences between $C D$ and MDD patients were also noted. CD patients had even lighter sleep than the depressed subjects, as seen in their significant increase in Stage 1 sleep and greater awake time. CD patients did not have elevated REM percentage as in the MDD patients, and did not have an elevation of REM activity or whole-night REM density.

Our sample of AICS patients is still small and the results are therefore preliminary. These four patients resembled the MDD patients even more closely than the CD patients did, in that they had very elevated total REM activity, had high REM density during the entire night as well as during the first REM period, and exhibited less abnormality in Stage 1 sleep percentage than CD patients. Further study of differences in the sleep of patients with CD and AICS should prove informative, as these two groups both demonstrate hypercortisolemia but differ in ACTH and beta lipotropin-beta endorphin levels, there being elevations in CD versus suppression in AICS.

Technical considerations may have had an influence on the results of this study. Because one clinical manifestation of CD and AICS is hyperarousal (Starkman et al 1981), it is thus possible that these patients may require a longer adaptation period than the customary single night. The AICS patients, however, were able to show a higher sleep efficiency than the $C D$ patients under the same procedure, suggesting that the pathophysiology of CD may be more important in explaining their poor sleep continuity. For both MDD and CS subjects, significant differences from normal subjects in percentage of $\delta$ sleep were not observed. This finding for CD patients is not consonant with the findings of Krieger and Glick (1974) or Krieger et al (1976), who reported clear decrements in $\delta$ sleep in CS patients. This may be due in part to the higher low-frequency filter settings in the normals $(0.5$ versus $0.3 \mathrm{~Hz}$ ), which could reduce $\delta$ activity and constitute a conservative 
bias against finding a difference between normal and patient groups. Further work would benefit from using computerized $\delta$ wave detection, which would increase the power to detect differences in $\delta$ sleep (Armitage et al 1992).

The hormonal and neural mechanisms underlying the similarities and differences of the abnormal sleep EEG patterns of patients with MDD, CD, and AICS are of theoretical interest. Because endocrine effects on, and interactions with, sleep stages are not yet fully understood, considerations of mechanisms can only be speculative at this time. However, the following neuroactive substances and anatomical areas may be involved in both MDD and CS.

Elevation and/or dysregulation of cortisol are common to MDD, CD, and AICS. Sleep continuity and maintenance were poor in patients with $C D$, suggesting that elevated cortisol may be associated with lighter sleep. Higher cortisol levels occurring toward morning in normal subjects have been associated with lighter sleep, in contrast to deeper sleep earlier in the nigit when cortisol is lower (Born et al 1986). Strong correlations between sleep continuity and cortisol levels were observed, as has been reported in MDD (Shipley et al 1989). Cortisol may have a role in slow wave sleep regulation, possibly acting through the mineralocorticoid (type l) receptor (Born et al 1991). This could account for some of the reduction in slow wave sleep reported by Krieger and Glick (1974).

Gillin et al (1972) found that administration of prednisone increased $\delta$ sleep and reduced REM sleep. Born et al (1991) attributed the suppression of REM sleep following cortisol infusion in healthy volunteers to binding specifically at the glucocorticoid (type II) receptor. CD patients, however, might differ in that there may be some adaptation to the chronic, very high levels of cortisol. In fact, our CD patients did not differ from normals in REM percentage.

The subgroup of CD patients with less elevated UFC had higher whole-night REM density and higher REM activity and density in the first REM period. Similarly, we found a negative correlation between baseline plasma cortisol levels and REM activity. This suggests that the extremely high cortisol production, and possibly the increased ACTH$B E$ levels, in some CD patients may be inhibitory to the production of REM sleep phasic activity. It should be noted, however, that in a preliminary assessment of MDD patients (Shipley et al 1989), REM activity was not associated with postdexamethasone cortisol level. Although our sample size is still too small to draw definitive conclusions, this suggests that the more highly elevated ACTH-BE in CD compared with MDD may be an important factor in this inhibition of phasic REM activity.

With regard to REM latency, Asnis et al (1983) demonstrated a negative correlation betweer. REM latency and baseline afternoon plasma cortisol level in MDD patients. Similarly, the prescnt sample of MDD patients came from a much larger group in which a negative correlation between REM latency and postdexamethasone plasma cortisol level in a $1 \mathrm{mg}$ DST was observed. In our sample of CD patients, REM latency, though significantly shortened, was not significantly correlated with baseline cortisol levels.

Other hormones and neuroactive substances are also likely of importance. Nocturnal growth hormone secretion is reduced in both MDD (Jarrett et al 1990) and CS (Krieger and Glick 1974), and this may be linked to shortened REM latency (Kupfer and Ehlers 1989). Delta-sleep-inducing peptide (DSIP), which is found in ACTH-containing pituitary cells (Bjartell et al 1989), may also be of significance. An important hormonal difference between MDD and Cushing's syndrome of both types is corticotropin-releasing hormone (CRH), which is suppressed in both CD and AICS yet is normal or elevated in MDD (Kalin et al 1986; Krishnan et al 1990). As our sample enlarges, sleep EEG differences 
between these subtypes of CS and MDD patients would suggest further support for the potential pathophysiological link between EEG sleep and arousal mechanisms and CRH suggested by Ehlers (1983).

The catecholamine system is influenced by corticosteroids. For example, cortisol stimulates tyrosine hydroxylase, the rate-limiting step in catecholamine synthesis (Schubert er al 1980). Steroid receptors located in the perikarya of central noradrenergic cells may influence genomic expression of these diffusely projecting systems which modulate the response of the organism to stress (Sulser 1989). In patients with CS, somatic symptoms of increased autonomic arousal such as increased sweating and restlessness are common, as are psychic symptoms of anxiety (Starkman et al 1981), and their plasma levels of norepinephrine are elevated (Cameron et al 1991). For patients with MDD, it has been suggested that their increased REM density and decreased slow wave sleep may reflect hyperarousal (Feinberg et al 1988; Feinberg and March 1988). Our findings of increased first REM period density in CD patients may be consistent with a hyperarousal hypothesis.

The hippocampus is a likely region mediating the effects of hypercortisolemia on sleep parameters in both CS and MDD. The hippocampus is associated with ciradian rhythms and is involved in the production of both REM and $\delta$ sleep (Melikov 1988). Animal research indicates that the hippocampus contains the highest concentration of cortisol binding sites in the brain, and that elevated levels of corticosteroids are toxic to subpopulations of these cells (Sapolsky et al 1988). Chronic exposure of the hippocampus to excessive levels of cortisol may therefore result in anatomic or functional alterations affecting sleep.

A final mechanism related to sleep alterations in CD and MDD patients is the newly appreciated interaction of HPA axis products such as cortisol and $\beta$ endorphin with lymphokines such as interleukin 1 or 2 . Corticosteroids have long been known to have immunosuppressant properties. In turn, lymphokines have significant effects on sleep and interact with the HPA axis (Krueger et al 1990).

We are currently enlarging our sample of patients with CD and AICS in order to investigate the association of sleep EEG abnormalities with HPA axis steroids and peptides, measures of immune function, and with the nature and severity of the affective and cognitive components of the depressive syndrome in these patients.

This work was supported by RO-1-MH43372 to Monica N. Starkman, NIAAA Center Grant 07378, NIH GCRC MOIRR00042, and a grant from NARSAD to Rajiv Tandon. The technical assistance of the GCRC nursing and laboratory staff is greatly appreciated.

\section{References}

Amsterdam JD, Winokur A, Abelman E, Lucki I, Rickels K (1983): Cosyntropin (ACTH alpha 1-24) stimulation test in depressed patients and healthy subjects. Am J Psychiatry 140:907909.

Armitage R, Roffwarg HP, Rush AJ, Calhoun S, Purdy D, Giles D (1992): Digital period analysis of sleep EEG in depression. Biol Psychiatry 31:52-68.

Asnis GM, Halbreich U, Sachar E, et al (1983): Plasma cortisol secretion and REM period latency in adult endogenous depression. Am J Psychiatry 140:750-753.

Bjartell A, Castro MG, Ekman R, Sundler F, Widerlov E, Loh YP (1989): Immunoreactive delta sleep-inducing peptide secretion from mouse dissociated, anterior pituitary cells: Regulation by corticotropin-releasing factor and arginine vasopressin. Neuroendocrinology 50:564-569. 
Born J, Kern W, Bieber K, Fehm WG, Schiebe M, Fehm HL (1986): Night-time plasma cortisol secretion is associated with specific sleep stages. Biol Psychiatry 21:1415-11424.

Born J, DeKloet ER, Wenz H, Kem W, Fehm HL (1991): Gluco- and antimineralocorticoid effects on human sleep: A role of central corticosteroid receptors. Am J Physiol 260(2 pt 1): E183E188.

Cameron OG, Starkman MN, Schteingart DE (1991): Catecholamines in Cushing's syndrome. Psychosom Med 53:245.

Carroll BJ, Feinberg M, Greden J, et al (1981): A specific laboratory test for the diagnosis of melancholia. Arch Gen Psychiartry 38:15-22.

Ehlers CL, Henriksen S, Wang M, Rivier J, Vale W, Bloom FE (1983): Corticotropin releasing factor produces increases in brain excitability and convulsive seizures in rats. Brain Res 278:332336.

Evans D, Nemeroff CB (1987): The clinical use of the dexamethasone suppression test in DSMIII affective disorders: Correlation with the severe depressive subtypes of melancholia and psychosis. J Psychiatr Res 21:185-194.

Feinberg I, March JD (1988): Cyclic delta peaks during sleep: Result of a pulsatile endocrine process? Arch Gen Psychiatry 45:1141-1142.

Feinberg, I, Baker T, Leder R, March JD (1988): Response of delta (0-3 Hz) EEG and eye movement density to a night with 100 minutes of sleep. Sleep 11:473-487.

Gillin JC, Jacobs LS, Fram DH, Snyder F (1972): Acute effect of a flucocorticoid on normal human sleep. Nature 237:398-399.

Haskett RF (1985): Diagnostic categorization of psychiatric disturbance in Cushing's syndrome. Am J Psychiatry 142:911-916.

Jarrett DB, Miewald MJ, Kupfer DJ (1990): Recurrent depression is associated with a persistent reduction in sleep-related growth hormone secretion. Arch Gen Psychiairy 47:113-118.

Kalin NH, Dawson G, Tariot P, et al (1986): Function of the adrenal cortex in patients with major depression. Psychiatry Res 22:117-125.

Krieger DT, Glick SM (1974): Sleep EEG stages and plasma growth hormone concentration in states of endogenous and exogenous hypercortisolemia or ACTH elevation. $J$ Clin Endocrinol Metab 39:986-1000.

Krieger DT, Howanitz PJ, Frantz AG (1976): Absence of noctumal elevation of plasma prolactin concentrations in Cushing's disease. $J$ Clin Endocrinoi Metab 42:260-272.

Krishnan KRR, Ritchie JC, Saunders WB, Nemeroff CB, Carroll BJ (1990): Adrenocorical sensitivity to low-dose ACTH administration in depressed patients. Biol Psychiatry 27:930-933.

Krueger JM, Toth L, Johannsen L, Opp MR (1990): Infectious disease and sleep: Involvement of neuroendocrine-neuroimmune mechanisms. Int J Neurosci 51:359-362.

Kupfer DJ, Ehlers CL (1989): Two roads to rapid eye movement latency. Arch Gen Psychiatry 46:945-948.

Melikiov EM (1988): Role of the hippocampus and its monoamine-reactive systems in the mechanisms of sleep in cats. Neuriasci Behav Physiol 18:9-16.

Rechtshaffen A, Kales A, Berger RJ (1968): A Manual of Standardized Terminology, Techniques, and Scoring System for Sleep Stages in Human Subjects. Public Health Service, U.S. Government Printing Office, Washington, DC.

Reynolds CF, Kupfer DJ (1987): Sleep research in affective illness: State of the art circa 1987. Sleep 10:199-215.

Sapolsky RM, Packan DR, Vale WW (1988): Glucocorticoid toxicity in the hippecampus: In vitro demonstration. Brain Res 21:367-371.

Schubert D, LaCorbiere, M, Klier F, Steinbach J (1980): The modulation of neurotransmitter synthesis by steroid hormones and insulin. Brain Res 190:67-79.

Shipley JE, Kumar A, Eiser A, et al (1989): The effect of in- or outpatient status on the association 
of EEG sleep data with post-dexamethasone cortisol levels in depressed patients. Sleep Res 18:185.

Shipley JE, Schteingart D, Tandon R, Starkman M (in press): Sleep architecture and sleep apnea in patients with Cushing's disease. Sleep.

Starkman MN (1987). Endocrinology and depression I: Hypothalamic-pituitary-adrenocortical hyperactivity and depression. In Cameron $\mathrm{O}$ (ed), Presentations of Depression New York: John Wiley, pp 251-273.

Starkman MN, Schteingart DE, Schork MA (1981): Depressed mood and other psychiatric manifestations of Cushing's syndrome: Relationship to hormone levels. Psychosom Med 43:3-18.

Starkman MN, Schteingart DE, Schork MA (1986): Cushing's syndrome after treatment: Changes in cortisol and ACTH levels, and amelioration of the depressive syndrome. Psychiatry Res 19:177-188.

Stokes PE, Stoll P, Koslow S, et al (1984): Pretreatment DST and hypothalamic-pituitary-adrenocortical function in depressed patients and comparison groups. Arch Gen Psychiatry 41:257267.

Sulser F (1989): New perspectives on the molecular pharmacology of affective disorders. Eur Arch Psychiatry Neurol Sci 238:231-239. 